\title{
Modified Widest Disjoint Paths Algorithm for Multipath Routing
}

\author{
Shangming Zhu ${ }^{1}$, Zhili Zhang ${ }^{2}$, and Xinhua Zhuang ${ }^{3}$ \\ ${ }^{1}$ Department of Computer Science, East China University of Science and Technology, \\ Shanghai, 200237, China \\ ${ }^{2}$ Department of Computer Science, University of Minnesota, Minneapolis, MN 55455, USA \\ ${ }^{3}$ Department of Computer Science, University of Missouri-Columbia, Columbia, \\ MO65211, USA \\ zhusm@ecust.edu.cn, zhzhang@Cs.umn.edu, zhuangx@missouri.edu
}

\begin{abstract}
Widest Disjoint Paths (WDP) algorithm is a promising multipath routing algorithm aimed at selecting good paths for routing a flow between a source-destination pair, where their bottleneck links are mutually disjoint. Nevertheless, the complexity of WDP algorithm is relatively high due to the fact that the good path selection process considers all available paths. To reduce its complexity, This paper proposes a modified WDP algorithm, which uses only a subset of available paths based on shortest widest paths, thereby limiting the number of candidate paths considered. As a result, the number of iterations in the good path selection process is significantly reduced. Performance analysis shows the modified scheme is more efficient than the original algorithm in a large network. Simulation results demonstrate that, in comparison with the original WDP algorithm, the modified WDP algorithm leads to lower latency and faster packets transferring process as the number of available paths increases.
\end{abstract}

Keywords: Widest disjoint paths, multipath routing, candidate path, width of path set.

\section{Introduction}

Multipath routing is designed to optimize the operational performance of a network by routing simultaneously along multiple paths on demand. It improves QoS (Quality of Service) of a network in using multiple "good" paths rather a single "best" path in routing. Several multipath routing algorithms have been proposed for balancing the load across a network [1-3]. WDP (Widest Disjoint Paths) [1,2] and LDM (Load Distribution over Multipath) [4] schemes make routing decisions at the flow level. The WDP algorithm is based on two concepts: path width and path distance. Path width is used to detect bottlenecks in the network and avoid them if possible. Path distance is indirectly dependent on the utilization ratio of each constituent link of the path. The $L D M$ algorithm attempts to find a minimal set of good paths based on two criteria: (a) the metric hop-count associated with each path kept as low as possible and (b) link utilization maintained inferior to a certain parameter. Schemes like 
ECMP (Equal Cost MultiPath) [5], MPLS-OMP (Optimized MultiPath) [6] and MATE (MPLS Adaptive Traffic Engineering) [7] perform packet level forwarding decisions. ECMP splits the traffic equally among multiple equal cost paths. But these paths are determined statically that may not reflect the congestion status of the network. It is always desirable to apportion the traffic according to the quality of each individual path. MPLS-OMP uses updates to gather link load information, selects a set of optimal paths and then distributes traffic among them. MATE does constant monitoring of links by using probe packets to measure link quality such as packet delay and packet loss.

The simulation results reported in [1] and [2] show that WDP is a promising multipath routing algorithm that can provide higher throughput and is capable of adapting to the changing network conditions at different time scales comparing to other schemes. In this paper, we attempt to improve the WDP algorithm in terms of computational efficiency.

\section{Widest Disjoint Path Problem}

The WDP algorithm is aimed at selecting good paths for routing a flow between a source-destination pair so that their bottleneck links are mutually disjoint. There are three tasks in the multipath routing: (a) dynamically select the candidate paths, (b) determine the good paths from the candidate paths and (c) proportion traffic among the good paths. This section lays out the basic assumptions for path selection.

In WDP algorithm, source routing is used and the network topology information is assumed available to all source nodes. One or multiple explicit-routed paths or label switched paths are set up statically between each source-destination pair using, e.g., MPLS. A flow is blocked when routed to a path whose bottleneck link has no bandwidth left.

Consider a network topology with $N$ nodes and $L$ links. Let $s$ be a source node and $d$ be a destination pair, and let $r$ denote a path in the network, i.e., $r$ is a set of links from a source to a destination. Let the maximum capacity of link $l$ be $\hat{\mathrm{C}}_{1}>0$, which is fixed and known in advance.

Let $\hat{R}$ denote the set of all available paths for routing flows between the source and destination node. The set of good paths $R$ is a subset of $\hat{R}$ selected by the good path selection algorithm for multipath routing. The WDP algorithm aims at computing a path set $R$ for routing a flow between a source-destination pair such that all paths in $R$ are mutually disjoint with regard to (w.r.t.) the bottleneck links.

\section{Modified WDP Algorithm}

The original WDP algorithm [1,2] compares all available paths against the width of the set of existing good paths for good path selection. To reduce its computation complexity, we modify the WDP algorithm in the following two aspects: (a) we use only a subset of available paths based on shortest widest paths rather than all available paths as the candidate paths; (b) we limit the number of candidate paths, hence, the number of iterations in good path computation. 


\subsection{Width of Path Set}

To determine whether a candidate path is a good one and whether to include it in the existing good path set, the width $W$ of a path set $R$ is calculated as follows.

First, the difference $C_{l}=\hat{\mathrm{C}}_{1}-v_{l}$ is defined as the average residual bandwidth on link $l$, where $v_{l}$ is the average load on the link. The width and distance of a path $r$ are defined as $\mathrm{w}_{\mathrm{r}}=\min _{\mathrm{l} \in \mathrm{r}} C_{l}$ and $\mathrm{d}_{\mathrm{r}}=\sum_{l \in r} 1 / C_{l}$, respectively. Disjoint paths in a path set are recursively determined w.r.t. the bottleneck links, and the sum of their widths is used to calculate the width of the path set.

Initially, $W$ is set to 0 . The width $w$ of each disjoint path $r$ is added to the width $W$ of path set $R$ according to their widths and distances. In each iteration, only the shortest widest paths are considered. A subset $R^{*}$ of paths with the widest width $w^{*}$ is identified. There may be more than one path with the widest width $w^{*}$, from these widest paths, a path $r^{*}$ with the shortest distance $d^{*}$ is selected and the width $w^{*}$ of shortest widest path $r^{*}$ is added to the total width $W$ by $W=W+w^{*}$.

To ensure the disjoint paths are only used to compute the width of set $R$, the residual capacities of all the links along each shortest widest path $r^{*}$ is reduced by an amount $w^{*}$ by $C_{l}=C_{l}-w^{*}$ and the path $r^{*}$ is removed from the set $R$. The above iteration process is repeated until the set $R$ becomes empty. The resulting $W$ is considered to be the width of $R$. The narrowest path, i.e., the last path removed from the set $R$, is referred to as $\operatorname{Narrowest}(R)$.

Based on the defined width of a path set, a new candidate path is added only if its inclusion increases the width of the set of good paths, and an existing good path is removed if its exclusion does not decrease the total width. When the number of good paths reaches a specified limit, a good path is replaced with another path if this change increases the width.

\subsection{Modified Good Paths Selection}

The success of WDP relies on how to efficiently select good paths. We observe that there is a significant overhead and high complexity in the original WDP algorithm because all available paths are considered. As the number of available paths increases, it becomes increasingly computational expensive to compute good paths.

To improve the efficiency of the original WDP algorithm and shorten the time for computing good paths, we use only a subset of available paths based on shortest widest paths, thereby reducing the number of iterations in good path computation. Our proposed good path selection algorithm is shown in Fig. 1.

First we exclude $R$ from $\hat{\mathrm{R}}$ to initiate candidate path set $A$, namely, $A=\hat{\mathrm{R}} \backslash R$ (excluding $R$ from $\hat{\mathrm{R}}$ ). Different from the original WDP algorithm, we set up a parameter $\eta_{0}$ as the maximum of iterations in this procedure to reduce the number of candidate paths and limit the number of iterations.

In each iteration, rather than consider all available paths, our proposed approach considers only a subset of available paths based on shortest widest paths to compute good paths, namely, it only selects a candidate path $r^{+}$with the widest width $w^{+}$and 


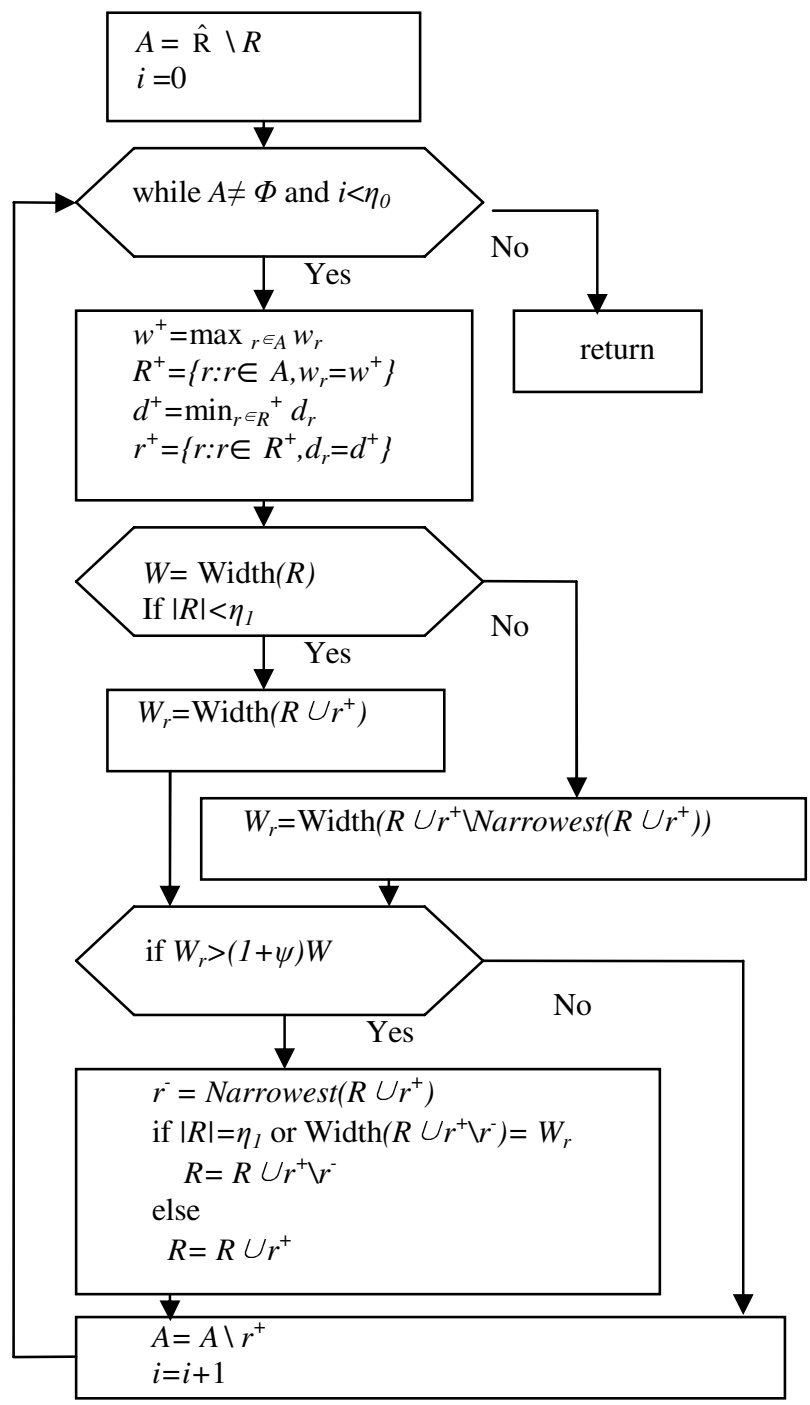

Fig. 1. Modified WDP algorithm

shortest distance $d^{+}$to compute good paths. It is possible that many candidate paths may have the same widest width $w^{+}$. Among such paths, the path $r^{+}$with the shortest distance $d^{+}$is chosen.

Second, the procedure of adding a new path to good path set and removing an existing good path from good path set or replacing it with another path is similar to the original WDP algorithm.

The width of a set of paths before inclusion $r^{+}$is defined as $W=\operatorname{Width}(R)$. The function Width() that computes width of a path set has been discussed in III.A. 
Inclusion of a candidate path $r^{+}$is determined based on the number of good path set $R$ and the width increase due to path $r^{+}$.

Depending on the number of multiple paths required by an application, a parameter $\eta_{1}$ is set up as the maximum number of good paths in WDP algorithm. If the number of good paths in the current good path set $R$ is below the specified limit $\eta_{1}$, the resulting width $W_{r}$ is the width after including $r^{+}$among $R$, i.e., $W_{r}=\operatorname{Width}\left(R \cup r^{+}\right)$; Otherwise, it is the width after including $r^{+}$but excluding the narrowest path among $R \cup r^{+}$, i.e., $W_{r}=\operatorname{Width}\left(R \cup r^{+} \backslash \operatorname{Narrowest}\left(R \cup r^{+}\right)\right)$.

This width $W_{r}$ is compared to the current width $W$ of the good path set. A candidate path is made a good one if its inclusion in set $R$ increases the width by a fraction $\psi$. Here $\psi>0$ is a configurable parameter to ensure that each addition improves the width by a significant amount.

If the width $W_{r}$ is more than $(1+\psi) W, r^{+}$will be added to the good path set. Before adding $r^{+}$, the path set $R \cup r^{+}$should be pruned, in other words, its narrowest path may be removed if needed using the following criterion Let $r^{-}$be the narrowest path in the set $R \cup r^{+}$. The path $r$ is replaced with $r^{+}$if either the number of good paths has already reached the limit $\eta_{1}$ or the path $r$ does not contribute to the width of the set. Otherwise, the path $r^{+}$is simply added to the set of good paths. If the width $W_{r}$ is not more than $(1+\psi) W$, no new path is added.

After each computation of candidate path set, the candidate path $r^{+}$is removed from $A$, and the number of iteration $i$ is modified. The iteration is repeated until $A$ is empty or its iteration reaches the maximum $\eta_{0}$.

\section{Performance Analyses}

\subsection{Analysis of Computational Efficiency}

It is obvious that the scheme described above always converges for all values of $\eta_{0}$ and $\eta_{1}$. The original WDP algorithm takes $O\left(n^{3}\right)$ iterations, where $n$ is the number of available paths between the pair of ingress-egress routers [3]. In contrast, the complexity of our proposed algorithm is $O\left(\eta_{0} \eta_{1} n\right)$, as selecting good paths from a set of $n$ paths depends on $\eta_{0}$ candidate paths and $\eta_{1}$ good paths. In general, $\eta_{0}$ and $\eta_{1}$ are far smaller than $n$, especially in a large network. Hence the modified scheme is more efficient than the original algorithm in terms of computational complexity.

\subsection{Simulation Results}

In this section the performance of the modified WDP algorithm is studied through simulation experiments using NS2. The simulation environment we use is shown in Fig. 2. There are two types of links: solid and dotted. To simplify the simulation, all solid links have the same capacity with $2 \mathrm{Mbps}$ of bandwidth and all the dotted links have the same capacity with $3 \mathrm{Mbps}$ of bandwidth. We also ignore the propagation delay of each links. There are 18 nodes and 30 links in the topology. To analyze the performance of our modified WDP algorithm, node 1 is chosen as the source node and node 18 is as the destination node. The default values for the WDP parameters are $\psi=0.2, \eta_{0}=3, \eta_{1}=2$. 
We use the end-to-end packet delivery latency (in short, latency) as a metric to evaluate the impact $\mathrm{f}$ the computational overheads of the modified WDP and the original WDP algorithm on the efficacy of multi-path routing. To measure the end-toend packet delivery latency, the packet sending rate at the source node is set to 4.5 Mbps.

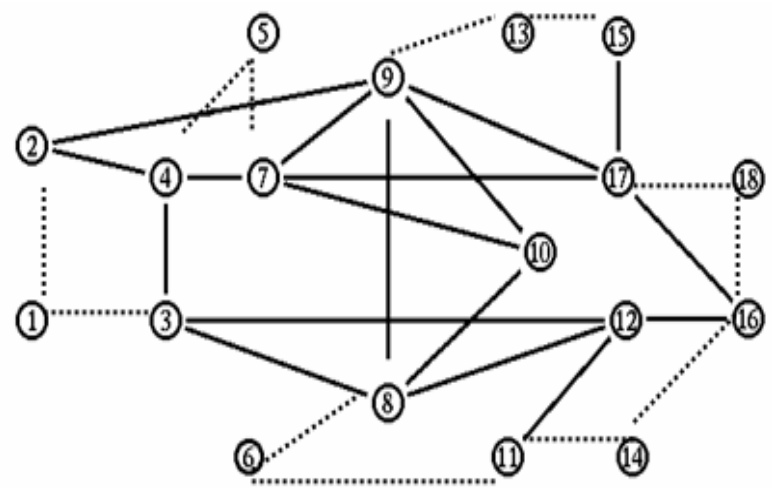

Fig. 2. Network model for the simulation

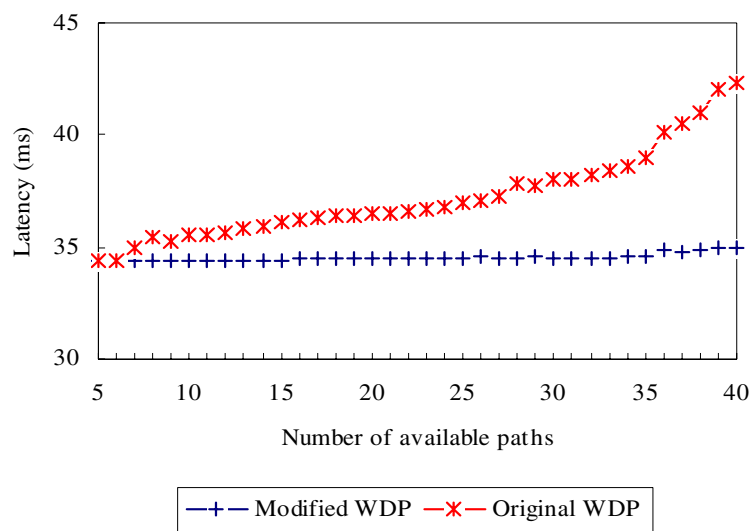

Fig. 3. Latency with variable numbers of available paths

Fig. 3 shows the latency variation as the number of available paths $n$ is varied. It is seen that as the number of available paths increases, the modified WDP performs better than the original WDP algorithm. When the number of available paths is small $(n \leq 6)$, there is not much difference of latency between the modified WDP and the original WDP algorithm; As the number of available paths increases, latency of the modified $W D P$ varies slightly and is much lower than that of the original WDP algorithm. 


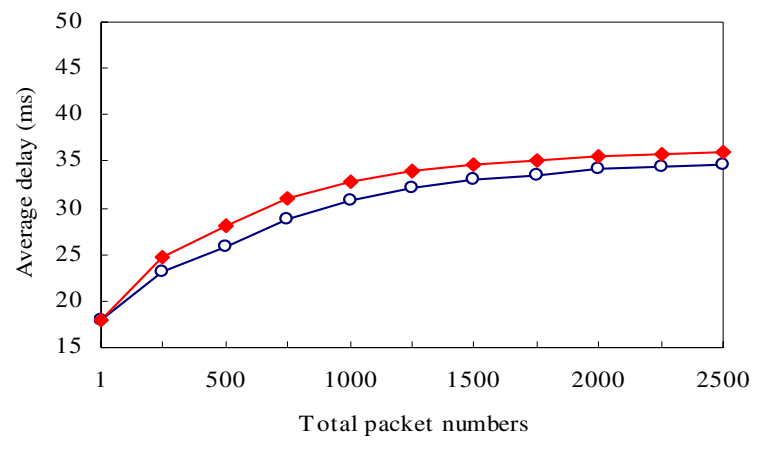

$\multimap-$ Modified WDP $\multimap-$ Original WDP

(a) $n=10$

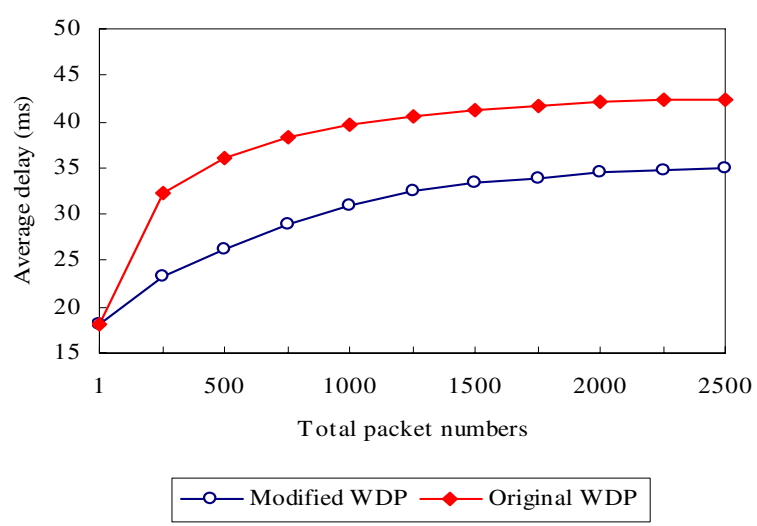

(b) $n=40$

Fig. 4. Comparison of average one way delay

Fig. 4 shows the comparison of the average one way delay using varying numbers of available paths. In Fig. 4(a) the number of available paths $n$ is set to 10; it is seen that the modified WDP is a little faster than the original WDP algorithm in the packets transferring process. In Fig. 4(b) $n$ is set to 40; it is seen that the modified WDP is much faster than the original WDP algorithm in the packets transferring process because of its better computational efficiency.

\section{Conclusions}

In this paper we propose an improvement to the Widest Disjoint Paths (WDP) routing algorithm which performs good paths selection based on the computation of the width of the disjoint paths with regard to bottleneck links. To reduce the complexity of this algorithm and shorten the time for computing good paths, we develop a modified $W D P$ algorithm by limiting the number of candidate paths and thereby reducing the 
number of iterations needed for good path computation. Our proposed approach only uses a subset of available paths based on shortest widest paths rather than all available paths to compute the good paths. Performance analysis shows the modified scheme is more efficient than the original algorithm in a large network. Simulation results demonstrate that the modified WDP algorithm leads to lower latency and faster packets transferring process as the number of available paths increases in comparison with the original WDP algorithm.

Acknowledgments. This work is supported in part by NIH, NSF, and the National Science Council (NSC) of Republic of China under grant number NIH DHHS 1 R01 DC04340-01A2, NSF EIA 9911095, and NSC-94-2213-E-006-068 respectively. This work was also supported by China Scholarship Council (CSC).

\section{References}

1. Nelakuditi, S., Zhang, Z.-L., Du, D.H.C.: On Selection of Candidate Paths for Proportional Routing. Computer Networks 44, 79-102 (2004)

2. Nelakuditi, S., Zhang, Z.-L.: On Selection of Paths for Multipath Routing. In: Wolf, L., Hutchinson, D.A., Steinmetz, R. (eds.) IWQoS 2001. LNCS, vol. 2092, pp. 170-184. Springer, Heidelberg (2001)

3. Lee, K., Toguyeni, A., Noce, A., Rahmani, A.: Comparison of Multipath Algorithms for Load Balancing in a MPLS Network. In: Kim, C. (ed.) ICOIN 2005. LNCS, vol. 3391, pp. 463-470. Springer, Heidelberg (2005)

4. Song, J., Kim, S., Lee, M.: Dynamic Load Distribution in MPLS Networks. In: Kahng, H.K. (ed.) ICOIN 2003. LNCS, vol. 2662, pp. 989-999. Springer, Heidelberg (2003)

5. Moy, J.: OSPF Version 2, RFC 2328. Internet Engineering Task Force (1998), http://www.ietf.org/rfc/rfc2328.txt

6. Villamizar, C.: MPLS Optimized Multipath (MPLS-OMP). Work in Progress InternetDraft, draft-villamizar-mpls-omp-01 (1999)

7. Elwalid, A., Jin, C., Low, S., Widjaja, I.: MATE: MPLS Adaptive Traffic Engineering. In: Proceedings of INFOCOM'2001, Alaska (2001) 